\section{Biggest COVID-19 trial tests repurposed drugs first}

The United Kingdom has launched the world's largest clinical trial to evaluate whether existing or new drugs work to treat people hospitalized with Covid-19. The Randomised Evaluation of COVID19 (RECOVERY) trial is led by University of Oxford researcher Peter Horby, Who previously worked with researchers in Wuhan to publish the first clinical data on the new coronavirus and previously led Ebola drug trials in the Democratic Republic of the Congo. It will follow an adaptive clinical trial design to accelerate the process of identifying effective treatments. Patients who test positive with COVID-19 who enter any of the United Kingdom's 132 National Health Service hospitals are invited to participate. Over 5,000 patients were enrolled in a month since the trial's launch in March.

Drugs included in the trial protocol are all existing medicines repurposed for COVID-19: AbbVie's Kaletra (lopinavir/ritonavir), commonly used to treat HIV infection; dexamethasone, an anti-inflammatory steroid; hydroxychloroquine, an antimalarial drug; and the antibiotic azithromycin. Trial participants are randomized to receive standard of care with one of the study drugs, or standard of care alone. The adaptive trial design allows the RECOVERY team to review the data regularly and quickly add or eliminate treatments, and to incorporate new treatments into the trial as they become available.

The UK-wide trial is supported by a grant to the University of Oxford from UK Research and Innovation and the National Institute for Health Research (NIHR) and by core funding provided by the NIHR Oxford Biomedical Research Centre, Wellcome, the government's Department for International Development, Health Data Research UK, the Medical Research Council Population Health Research Unit, NIHR Clinical Trials Unit support funding and the Bill and Melinda Gates Foundation. The foundation has pledged more than $\$ 250$ million to the global COVID-19 response, in particular to support African and South Asian countries to roll out detection, treatment and isolation programs.

Published online: 11 May 2020 https://doi.org/10.1038/s41587-020-0528-X for patients, M2-like TAMs typically predominate in most tumors.

Aiming to reprogram those TAMs, more than two dozen companies are now developing drugs directed against the CD47 axis. The best known and most clinically advanced of these is Forty Seven, maker of a humanized IgG4 monoclonal antibody called magrolimab. This CD47-targeted therapy is slated to enter registration-enabling trials in the coming months and could reach the market as soon as 2022. In March, Gilead Sciences announced plans to pay $\$ 4.9$ billion for Forty Seven, an acquisition that scientists involved with Carisma and Myeloid both point to as a financial stamp of approval on the strategy of macrophage modulation more broadly.

"There is increasing interest in macrophages in the immuno-oncology field," says Saar Gill, a University of Pennsylvania Perelman School of Medicine hematologist who cofounded Carisma together with his former graduate student Michael Klichinsky. Drugs like magrolimab come with many challenges, however, including the need to preferentially target M2-like macrophages in the tumor microenvironment over others distributed throughout the body. "CAR-macrophages are intrinsically different," notes Klichinsky - which helps explain why engineered macrophage strategies like Carisma's are gaining more attention.

For the company's lead program, CT-0508, Gill and Klichinsky (together with cell therapy pioneer Carl June, who co-advised Klichinsky during his $\mathrm{PhD}$ and now serves as a scientific advisor to Carisma) took advantage of a chimeric adenoviral vector called Ad5f35 to transduce primary human macrophages with a first-generation CAR construct of the kind evaluated clinically more than a decade ago in adoptive T-cell therapies. The CAR comprises an extracellular single-chain antibody variable fragment against HER2, a CD8 hinge and transmembrane domain, and a $\mathrm{CD} 3 \zeta$ intracellular signaling domain. (Later-generation CARs, including those found in Novartis's Kymriah (tisagenlecleucel) and Kite Pharma's Yescarta (axicabtagene ciloleucel) - the only two marketed CAR-T cell therapies for blood cancers - have included additional costimulatory signaling domains such as CD28 or 4-1BB.)

In their March paper, the researchers reported that the gene transfer method worked with about $75 \%$ efficiency. But according to Klichinsky, now vice president of discovery research at Carisma, "we can now get close to $100 \%$ transduction with our optimized protocols."
That high level of transduction could prove critical for clinical translation, says Weiskopf. Unlike T cells, which undergo massive clonal expansion in laboratory culture, monocyte-derived macrophages do not proliferate ex vivo. "So, what you put in is basically what you get out," he says. "That presents a hurdle in terms of the number of cells you have to start with. But if you make something that's very efficient, then potentially you can get by with a lower cell count to begin with."

Klichinsky is confident the protocols developed for CT-0508 can be scaled to produce the billions of CAR-macrophages needed for therapeutic purposes. And, as he points out, Carisma's Ad5f35 vector serves as more than just a conduit for efficient CAR delivery. The vector's assault on the cells, needed to introduce the genetic material, also spurs an antiviral response from the macrophages, leading them to adopt a durable M1 phenotype that, for unknown reasons, locks them into a proinflammatory state.

This leads to "a two-in-one effect during transduction," Klichinsky says. The HER2-directed CAR-macrophages not only carry out antigen-specific phagocytosis and tumor clearance, both in cell culture and in xenograft mouse models, but they also express proinflammatory factors in the tumor microenvironment that convert bystander TAMs to M1 macrophages, while promoting T-cell co-stimulation and recruitment. Although the researchers performed some confirmatory studies in mice transplanted with human hematopoietic stem cells, further testing in syngeneic models - and ultimately in patients - will be needed to show whether these mechanisms are active and can effectively eliminate antigen-expressing cancers without on-target, off-tumor toxicities, notes Weiskopf.

Prior to the Carisma team's paper, a team led by Ron Vale, a cell biologist at the University of California, San Francisco (UCSF), had already shown that CAR-expressing mouse macrophages could drive antigen-directed engulfment of whole cancer cells. But his group had not looked much beyond phagocytosis to other macrophage functions, such as the ability of engineered cells to remodel the tumor microenvironment or to kill tumor cells in mice. "That is what I was most excited to see" in the Carisma report, says Meghan Morrissey, a postdoc in Vale's lab who co-led the prior UCSF study. "This creates a signal amplification mechanism" unlike any seen with other types of adoptive cell therapies.

Another notable difference between the UCSF and Carisma projects was the choice 